\title{
Long-Term Follow-Up of Children, Adolescents, and Young Adult Cancer Survivors
}

\author{
Inken Hilgendorf ${ }^{a} \quad$ Corinna Bergelt ${ }^{b}$ Carsten Bokemeyer ${ }^{c}$ Peter Kaatsch ${ }^{d}$ \\ Ulf Seifart $^{\mathrm{e}}$ Alexander Stein $^{\mathrm{f}}$ Thorsten Langer ${ }^{\mathrm{g}}$ \\ ${ }^{a}$ Klinik für Innere Medizin II, Abteilung für Hämatologie und Onkologie, Universitätsklinikum Jena, Jena, Germany; \\ b Zentrum für Psychosoziale Medizin, Institut und Poliklinik für Medizinische Psychologie, Universitätsklinikum \\ Hamburg-Eppendorf, Hamburg, Germany; ' CHubertus Wald Tumor Zentrum, II. Medizinische Klinik und Poliklinik, \\ Universitätsklinikum Hamburg-Eppendorf, Hamburg, Germany; ${ }^{d}$ Deutsches Kinderkrebsregister am Institut für \\ Medizinische Biometrie, Epidemiologie und Informatik (IMBEI), Universitätsmedizin der Johannes-Gutenberg- \\ Universität Mainz, Mainz, Germany; ${ }^{\text {}}$ Klinik für Hämatologie und Onkologie, Klinik Sonnenblick der Deutschen \\ Rentenversicherung Hessen, Marburg, Germany; ${ }^{f}$ Hämatologisch-Onkologische Praxis Hamburg-Eppendorf, \\ Hamburg, Germany; ${ }^{9}$ Klinik für Kinder- und Jugendmedizin, Universitätsklinikum Schleswig-Holstein, Campus \\ Lübeck, Lübeck, Germany
}

\section{Keywords}

AYA cancer survivor · Childhood cancer survivor · Late effects · Long-term follow-up

\begin{abstract}
Background and Summary: Thanks to increasing cure rates to currently $>80 \%$, children, adolescents, and young adults (CAYA) survive their cancer much more frequently today than decades ago. Due to their long life expectancy, CAYA cancer survivors are at a particular risk of long-term sequelae from the cancer itself or the therapy applied; this requires specific follow-up, and preventative or even therapeutic interventions. Thus, compared to the normal population, morbidity and mortality may be significantly increased. In 2 of 3 survivors, the cancer and the respective treatment can lead to late effects, even after 30 years, which require specific therapy; in about one-third of these cases, these effects are classed as severe. Applying structured follow-up could identify these late effects at an early stage and initiate immediate treatment. In 2018, a working group dealing with long-term survival after cancer detected $<40$ years of age was founded within the framework of the National Cancer Plan of the German Federal Ministry of Health.

(c) 2021 S. Karger AG, Basel
\end{abstract}

\section{Introduction}

Each year in Germany, about 1,800 children, 360 adolescents, and 16,500 young adults $\leq 39$ years) (CAYA) receive a diagnosis of malignant hematological or oncological disease [1]. This heterogeneous age cohort constitutes only a minority of cancer patients. With current cure rates of $>80 \%$, cancer survival is far better than 40 years ago. Depending on the respective cancer type, CAYA are treated whenever possible according to optimization studies provided by the GPOH (Gesellschaft für pädiatrische Onkologie und Hämatologie), the GSHG (German Hodgkin Study Group) or the GMALL (German Multicenter Study Group for Adult Acute Lymphoblastic Leukemia) [2, 3]. Accordingly, data on disease, treatment, and follow-up are collected

This paper reflects the considerations of this working group and aims to define the need to offer needs-oriented follow-up and support for CAYA following cancer treatment, based on the latest developments and taking existing structures into consideration. In addition, the statement will highlight measures to optimize current CAYA survivor care by establishing suitable follow-up and surveillance structures and facilities. 
with the consent of patients, or, in the case of minors, their parents.

Cancer survivors are subject to long-term sequelae, from either the cancer itself or the therapy applied, that require specific follow-up as well as preventative or even therapeutic interventions. In about 2 of 3 survivors, the cancer and the respective treatment can lead to late effects, even after 30 years [4]. Overall, AYA cancer survivors were found to have a 10.4-fold increased risk of death than a matched noncancer cohort, and this risk remained elevated $>20$ years after diagnosis [5]. In addition, the incidence of second cancer-related mortality exceeded the rate of recurrence-related mortality, starting 15 years after diagnosis, and similar trends have been observed for death from other health-related conditions [5]. Infections and cardiovascular and renal diseases are the most common noncancer causes of mortality; the highest standardized mortality ratios (SMRs) were reported following treatment for non-Hodgkin lymphoma (SMR = 6.33) or leukemia $(S M R=5.26)$ [6]. Even 20 years after a cancer diagnosis, an increased SMR not caused by the initial cancer was identified. These US data highlight the need for risk-adapted (depending on the type of cancer and therapy), qualified, and structured long-term follow-up for all cancer survivors, that takes into account all aspects of physiological and psychosocial late effects. Some of the secondary diseases known to date are listed in the info box "Long-term morbidity in CAYA cancer survivors."

Although registers are in place to record late effects in some patients (e.g., after cancer during childhood), the coverage in Germany is very limited, particularly for young adult cancer survivors. The successful implementation of structured survivorship programs, mainly at academic centers, show the feasibility and high acceptance by CAYA cancer survivors. Of particular note is the prevention program, Comprehensive Assessments and Related Interventions to Enhance Long-Term Outcome in Children, Adolescents and Young Adults (CARE for CAYA), which is offered to young cancer survivors aged 15-39 years at 14 centers across Germany. However, due to the lack of reimbursement by health insurance companies, these programs rely completely on third-party financing (e.g., CARE for CAYA by a grant from the Federal Joint Committee, limited to 3 years) [8-10]. Furthermore, there is no broadly available needs assessment or established risk stratification system available in Germany.

\section{Therapy Optimization Studies and Research into Late Effects}

Alongside the optimization of therapy and higher cure rates, as early as the 1980s, studies were being conducted that focused on reducing late effects. Of particular interest

\section{Box}

Long-term morbidity in CAYA cancer survivors

Bones/joints/musculature/bone metabolism

Endocrine disorders

Cardiovascular disorders

Secondary tumors/malignancies

Central nervous system (CNS)/peripheral nervous system disorders

Renal and urinary tract disorders

Disorders of the sensory organs (i.e., eyes and ears)

Pulmonary disorders

Gastrointestinal disorders

Skin/hair/tooth disorders

Immune system disorders

Long-term psychosocial effects

Other aspects (see also [7])

is the reduction/elimination of radiotherapy and/or cytostatic drugs in the treatment of Hodgkin lymphoma, and of cranial irradiation in the treatment of acute leukemia in children [11-13]. In acute leukemia, improved risk stratification enabled treatment reduction for patients with a lower risk of recurrence [14], particularly via the diagnosis of minimal residual disease (MRD) [15]. The results of research into late effects are therefore continuously affecting first-line treatment de-escalation strategies.

Thus, survival rates of CAYA by age, tumor stage, gender, and treatment, in addition to late and long-term sequelae, must be recorded in a comprehensive and structured way. The fundamental prerequisite is improved networking between all follow-up institutions, including all existing registers and databases (e.g., the German Child Cancer Register [GCCR], the Federal Cancer Registries, the German Stem Cell Transplant Register [DRST Register], the German Pharmacoepidemiological Research Database [GePaRD], etc.) as well as the consecutive development of a common registry or data platform. In addition, the development of risk stratification based on the systematic analysis of existing registry data, epidemiological surveys, and available molecular profiling data is required. This risk stratification system will be applied to obtain individualized needs-based follow-up recommendations and establish preventative care options that incorporate the characteristics of patients, diseases, and treatments.

\section{The Role of the GPOH and DGHO}

Section 2 of the statutes of the GPOH (29/05/2010) and DGHO (30/09/2018) define the roles of these associations as promoting experimental, clinical, hematologi$\mathrm{cal}$, and oncological research as well as providing education and care. Surveillance, follow-up, and the provision of follow-up structures belong to this mission. In the 
Roadmap 2019 for Research into Cancer and Blood Disorders in Germany published by the DGHO [16], the recording and analysis of long-term survival with and after cancer is defined as one of its research priorities. Since the late 1980s, the GPOH has been the mainstay for the creation of follow-up structures that, together with therapy optimization studies, has advanced the research into late effects in children and adolescents. Nonetheless, the registries for the collection of late effects, particularly in adult cancer survivors, and thus the available data, remain incomplete.

\section{Terms and Definitions}

The classification of cancer survivorship, with specific focus on long-term survival, is phase-dependent, i.e., acute ( 1 year), prolonged ( $2-5$ years), and long-term ( $>5$ years).

\section{Information about Follow-Up Options}

Information about local follow-up options following CAYA cancer therapy (CAYA survivor programs) is available to former patients and their families in Germany at the following websites:

- www.kinderkrebsinfo.de/nachsorgeangebote

- https://junge-erwachsene-mit-krebs.de/jung-undkrebs/angebote-in-deiner-naehe/

\section{Procurement Sources for Results of Long-Term Surveillance}

The GCCR's surveillance findings and the multiple national and international studies $[6,17]$ show an urgent need for structured follow-up. This is especially relevant for the specific problems of CAYA cancer survivors, which are substantially different from the medical and psychosocial problems of cancer survivors in later adulthood. Many former patients express the desire for structured, long-term follow-up, as there is often a lack of adequate care options.

The continuous and prospective data collection of the cancer stage at the time of diagnosis and the long-term course of the disease, depending on therapy methods and modalities, is necessary to ascertain the care needs of CAYA cancer survivors and to create appropriate treatment approaches. A computer-based risk stratification system with algorithms based on the "therapeutic/tumorrelated" risk profile, lifestyle, and family-related risk factors should be established. With the help of a continuous survey, the current risk should be determined regularly, followed by referral of the CAYA cancer survivor to the respective care structure. In some cases, allocation to a specialized local aftercare center or a special prevention program may be required.
Structured long-term surveillance is important for evaluating the impact of therapy optimization studies on the incidence and relevance of late effects. The surveillance can be carried out by either requesting findings from follow-up consultations or by means of a direct patient questionnaire.

\section{Interdisciplinary Follow-Up Consultations/Centers for} CAYA

Follow-up consultations for children and adolescents with cancer are available at all pediatric oncology centers in Germany, Austria, and Switzerland. However, there is a paucity of data regarding late effects after CAYA cancer treatment, due to the absence of structured and riskstratified aftercare concepts. Therefore, all CAYA cancer survivors should receive a treatment summary and an individualized and risk-adapted follow-up care plan. A new care structure that is currently being developed for adults who had cancer in the CAYA age range involves interdisciplinary transition or follow-up consultations characterized by the close cooperation of pediatric oncologists, internal medicine specialists/hematologists, oncologists and other specialist disciplines, as well as psychosocial services [18]. This should also work together with qualified rehabilitation facilities for young adults and patients' associations, or self-help groups. This needs to be gauged on an individual basis at each location, and networking needs to take place between the specialists on site in the clinic and in the registered doctors' sector.

Ideally, research and health care projects (health care research) are defined and carried out as part of the routine follow-up process. Close collaboration with treating physicians and general practitioners is essential.

\section{Research Projects and Patient Questionnaires}

The GPOH's "Long-Term Effects" research committee evaluates projects by means of patient questionnaires after tumor therapy in childhood and carries this out through the GCCR together with the project group. Furthermore, the DGHO's AYA network working group provides a platform that enables multicenter research projects into late effects after tumor therapy. The surveillance carried out is dependent on the underlying disease and the nature and intensity of the tumor therapy, according to health care needs and intensity with both approaches.

\section{Data and Information Exchange}

Data specific to particular working groups and projects are collected as part of late effects research. The aim is to link these different data sets together in the future 
and allow them to be used interchangeably according to defined rules. The linking of all follow-up data collected by the various research and working groups into a single follow-up/late effects database (or in a decentralized way through defined interfaces or virtual databases) is a desirable goal. In addition, biobanks are used to support the scientific investigation of late and long-term effects following cancer and cancer therapy.

These structures and structural measures are crucial for long-term surveillance. Projects for working with scientific questions in the long-term survivor cohort should be linked to data entries from structured routine followups after cancer treatment. However, cooperation agreements must be drawn up in writing for scientific research projects. Structural provision measures, such as the logistics of questionnaires for patients or the preparation of therapy data, can be settled with a flat fee in certain cases. The promotion of structure-defining and structuremaintaining measures is an important contribution to quality assurance.

\section{Inclusion of Health Economists in the Optimization of Follow-Up for CAYA Who Have Previously Had Cancer}

The aim of including health economists and health care researchers is to evaluate the costs of late effects and interventions in collaboration with German health insurance funds, in order to lay the foundations for a late-effects health care option (e.g., interdisciplinary follow-up consultation, "survivor consultation"). Late effects/secondary diseases are associated with different direct and indirect, tangible and intangible, and individual and social costs. To ensure the sustainability of such a health care option, responsibility for financing also needs to be justified by social solidarity and social protection systems. A prerequisite for social sustainability is that the benefits of such an option must be verified and it must be possible to implement the health care option effectively and economically. This aim is based on the hypothesis that a professional health care option for late effects can avoid, or at least mitigate, the negative consequences of late effects following cancer in the CAYA age range as well as avoid or reduce the resulting costs, and that the additional social and individual benefits that it can achieve justify the costs associated with such an option.

Furthermore, the reappraisal of the issue of late effects also has an impact on first-line therapy. For example, in cases of acute leukemia, the indication of radiotherapy to the brain in children was significantly reduced after the observed accumulation of secondary brain tumors as well as cognitive and endocrinological deficits.

\section{Existing Principles}

- It is essential to establish survivorship programs for the prospective, structured recording of long-term effects following tumor therapy, as well as needsbased coverage with interdisciplinary follow-up consultations/centers, for CAYA who previously had cancer and are now adults, so as to determine the need for prevention and treatment and the riskadapted allocation of resources. The interdisciplinary follow-up of CAYA survivors who have previously had cancer should be carried out in accordance with guidelines.

- To obtain more information on the state of health of former patients, in addition to the answers in the patient questionnaire, a status request is also desirable in the abovementioned follow-up consultations for CAYA who have previously had cancer. These data should then be transferred to the GCCR or the epidemiological state cancer register to complete the historical data.

- For the purposes of health care research, the issue of health economics should be dealt with, along with diagnostic and therapeutic issues in follow-up, and the effectiveness of care structures for CAYA long-term survivors should be analyzed.

- The linking of all follow-up data from the various research and working groups via defined interfaces between the existing follow-up/late effects databases should be established.

- The structured establishment of supraregional biobanks, for researching the age-dependent molecular fundamentals of cancer on the one hand, and for systematically analyzing the long-term effects of tumor therapy on the other, is necessary.

- Fundamentals adopted by the executive board of the GPOH $[19,20]$ were: personal contact with survivors only via one and the same institution (the GCCR), avoid uncoordinated contact, ensure regular contact, and have an overview of the surveys in which the individual survivor had participated. These fundamentals as well as the objectives prioritized in 2019 in the DGHO's research roadmap continue to apply.

\section{Steps to Improve Long-Term Follow-Up Care}

\section{Survivorship Care Plan}

Each CAYA cancer survivor should receive (1) a treatment summary including their cancer history, (2) a riskadapted survivorship care plan, and (3) an age-adapted education about the risk of long-term sequelae, the necessity of appropriate aftercare, and the relevance of a healthy lifestyle. 


\section{Specialized Rehabilitation Programs and Financial} Support for CAYA Cancer Survivors

Cancer-related financial distress is especially high in CAYA cancer survivors. In Germany, every fourth patient who returned to work and 2 of 3 cancer survivors not returning to work reported cancer-related financial distress [21]. Therefore, participation in peer group- or family-oriented specialized rehabilitation programs should be offered proactively, to improve the physical and psychosocial performance of cancer survivors and enable them to return to employment. In addition, targeted financial support (e.g., payment of a bridging allowance and exemption from out-of-pocket payment) and social counseling for CAYA cancer survivors in need may help them to overcome the hurdle of financial distress.

\section{Transition and Access to Specialized Aftercare Services for CAYA Cancer Survivors}

For childhood cancer survivors in Germany, a few multidisciplinary late-effects clinics [18] as well as transition programs have already been established. However, to ensure risk-adapted, long-term follow-up care, a nationwide transition program from pediatric to adult health care should be established. In addition, all CAYA cancer survivors should have access to a network of specialized aftercare services or survivorship clinics to reduce the burden of chronic health conditions after cancer treatment and ensure appropriate, standardized, and structured surveillance for late effects.

\section{Conclusion}

The GPOH's long-term follow-up working group and the AYA network working group form the central discussion and presentation platform for the professional asso- ciations within long-term surveillance for all interested physicians (pediatric oncologists, internal medicine specialists, hematologists, oncologists, radiotherapists, etc.), psychologists, practitioners of social medicine, rehabilitation clinics, researchers, patient/self-help groups, parents' and advocacy groups, foundations etc. Investigations during follow-up particularly relate to avoiding, recording, or researching late effects. They can be carried out across multiple studies, or be specific to one study, and can include structural provision measures (quality assurance) or scientific research projects.

In structured long-term surveillance supported by a multidisciplinary team, the needs of the survivor must be consistently met and the survival quality of the treatment documented.

\section{Conflict of Interest Statement}

The authors have no conflicts of interest to declare.

\section{Funding Sources}

A.S., C.B., I.H., and T.L. received research funding for the Care for CAYA program. The program is financed by the innovation fund of the German Federal Joint Committee (GBA). The funders played no role in the decision to submit the manuscript for publication.

\section{Author Contributions}

T.L. and I.H. drafted the manuscript. All authors provided comments and critical revisions. The final version was approved by all authors prior to submission.

\section{References}

1 Gesellschaft Der Epidemiologischen Krebsregister in Deutschland E.V. Krebs in Deutschland 2015/2016. Berlin: Robert KochInstitut; 2019.

2 Eichenauer DA, Plütschow A, Fuchs M, Sasse S, Baues C, Böll B, et al. Long-Term FollowUp of Patients With Nodular LymphocytePredominant Hodgkin Lymphoma Treated in the HD7 to HD15 Trials: A Report From the German Hodgkin Study Group. J Clin Oncol. 2020 Mar;38(7):698-705.

3 Rossig C, Juergens H, Schrappe M, Moericke A, Henze G, von Stackelberg A, et al. Effective childhood cancer treatment. http://onlinelibrary.wiley.com/doi/ 10.1002/pbc.24598/pdf

4 Armstrong GT, Liu Q, Yasui Y, Huang S, Ness KK, Leisenring W, et al. Long-term outcomes among adult survivors of childhood central nervous system malignancies in the Childhood Cancer Survivor Study. J Natl Cancer Inst. 2009 Jul;101(13):946-58.

5 Armenian SH, Xu L, Cannavale KL, Wong FL, Bhatia S, Chao C. Cause-specific mortality in survivors of adolescent and young adult cancer. Cancer. 2020 May;126(10):2305-16.

6 Anderson C, Lund JL, Weaver MA, Wood WA, Olshan AF, Nichols HB. Noncancer mortality among adolescents and young adults with cancer. Cancer. 2019 Jun;125(12): 2107-14.

7 Hudson MM, Ness KK, Gurney JG, Mulrooney DA, Chemaitilly W, Krull KR, et al. Clinical ascertainment of health outcomes among adults treated for childhood cancer. JAMA. 2013 Jun;309(22):2371-81.
8 Koch B, Salchow J, Grundherr J von, Elmers S, Escherich G, Rutkowski S, et al. Comprehensive assessments and related interventions to enhance long-term outcome in children, adolescents and young adults.

9 Quidde J, Koch B, Salchow J, Jensen W, von Grundherr J, Escherich G, et al. [Internet] Das CARE-for-CAYA-Programm. Available from: https://doi.org/10.1007/s12312-0170340-9.

10 Salchow J, Mann J, Koch B, von Grundherr J, Jensen W, Elmers S, et al. Comprehensive assessments and related interventions to enhance the long-term outcomes of child, adolescent and young adult cancer survivors presentation of the CARE for CAYA-Program study protocol and associated literature review. BMC Cancer. 2020 Jan;20(1):16. 
11 Löning L, Zimmermann M, Reiter A, Kaatsch P, Henze G, Riehm H, et al. Secondary neoplasms subsequent to Berlin-FrankfurtMünster therapy of acute lymphoblastic leukemia in childhood: significantly lower risk without cranial radiotherapy. Blood. 2000 May;95(9):2770-5.

12 Reinhardt D, Thiele C, Creutzig U; AMLBFM-Studiengruppe. Neuropsychologische Folgen der prophylaktischen ZNS-Bestrahlung bei Kindern mit akuter myeloischer Leukämie. Klin Padiatr. 2002 Jan-Feb;214(1):229.

13 Schellong G, Riepenhausen M, Creutzig U, Ritter J, Harbott J, Mann G, et al.; GermanAustrian Pediatric Hodgkin's Disease Group. Low risk of secondary leukemias after chemotherapy without mechlorethamine in childhood Hodgkin's disease. J Clin Oncol. 1997 Jun;15(6):2247-53.
14 Vora A, Goulden N, Wade R, Mitchell C, Hancock J, Hough R, et al. Treatment reduction for children and young adults with lowrisk acute lymphoblastic leukaemia defined by minimal residual disease (UKALL 2003): a randomised controlled trial. Lancet Oncol. 2013 Mar;14(3):199-209.

15 Conter V, Bartram CR, Valsecchi MG, Schrauder A, Panzer-Grümayer R, Möricke A, et al. Molecular response to treatment redefines all prognostic factors in children and adolescents with B-cell precursor acute lymphoblastic leukemia: results in 3184 patients of the AIEOP-BFM ALL 2000 study. Blood. 2010 Apr;115(16):3206-14.

16 DGHO. Roadmap 2019 für die Erforschung von Krebs- und Bluterkrankungen in Deutschland; 2019.

17 Hjorth L, Haupt R, Skinner R, Grabow D, Byrne J, Karner S, et al.; PanCare Network. Survivorship after childhood cancer: PanCare: a European Network to promote optimal long-term care. Eur J Cancer. 2015 Jul; 51(10):1203-11.
18 Gebauer J, Rieken S, Schuster S, Hahn B, Gebauer N, Meidenbauer N, et al. Multidisciplinary Late Effects Clinics for Childhood Cancer Survivors in Germany - a Two-Center Study. Oncol Res Treat. 2018;41(7-8):430-6.

19 Calaminus G, Kaatsch P. Positionspapier der Gesellschaft für Pädiatrische Onkologie und Hämatologie (GPOH) zu (Langzeit-)Nachbeobachtung, (Langzeit-)Nachsorge und Spätfolgenerhebung bei pädiatrisch-onkologischen Patienten. Klin Padiatr. 2007 MayJun;219(3):173-8.

20 Langer T, Grabow D, Kaatsch P, Creutzig U, Eggert A, Escherich G, et al. Langzeitbeobachtung ehemaliger krebskranker Kinder und Jugendlicher. Klin Padiatr. 2018 Oct;230(6): 291-8.

21 Leuteritz K, Friedrich M, Sender A, Richter D, Mehnert-Theuerkauf A, Sauter S, et al. Return to Work and Employment Situation of Young Adult Cancer Survivors: Results from the Adolescent and Young Adult-Leipzig Study. J Adolesc Young Adult Oncol. 2020. DOI: 10.1089/jayao.2020.0055 